\title{
Classification of Electrocardiogram Signals with RS and Quantum Neural Networks
}

\author{
X. Tang ${ }^{1}$ and L. Shu ${ }^{2}$ \\ ${ }^{1}$ School of Mathematical Sciences, \\ University of Electronic Science and Technology of China \\ Qingshuihe Campus of UESTC, No.2006, Xiyuan Avenue, West Hi-tech Zone, \\ Chengdu, Sichuan, P.R.China Zip/Postal Code:611731 \\ ${ }^{2}$ School of Mathematical Sciences, \\ University of Electronic Science and Technology of China \\ Qingshuihe Campus of UESTC, No.2006, Xiyuan Avenue, West Hi-tech Zone, \\ Chengdu, Sichuan, P.R.China Zip/PostavCode:611731 \\ 80651177@163.com,lanshu@uestc.edu.cn

\section{Abstract}

In this paper, rough sets $(R S)$ and quantum neuralnetwork $(Q N N)$ are used to recognize electrocardiogram (ECG) signals. Firstly wavele transform (WT) is used as a feature extraction after normalization of these) stgnals. Then the attribute reduction of RS has been applied as preprocessor so that we could delete redundant attributes and conflicting objects from decision making table but remain efficient information lossless. We realized classification modeling and forecasting test based on QNN after that. Finally, the RS-QNN gives us fast and realistic results compared with the BP and RBF. By this method, we could reduce the dimension of feature space and decrease the complexity in the process. Experiment result shows that the classifteatign ability of the RS-QNN is superior to conventional approach.

Keywords: Rough Sets, Quantum Neural Network, Electrocardiogram, Wavelet Transform

\section{Introduction}

In recent years, the automatic classification of electrocardiogram (ECG) signals has received great attention from the biomedical engineering community. Electrocardiography is an important tool in diagnosing the condition of the heart. The electrocardiogram (ECG) is the record of variation of bioelectric potential with respect to time as the human heart beats. It provides valuable information about the functional aspects of the heart and cardiovascular system. Early detection of heart diseases can prolong life and enhance the quality of living through appropriate treatment. Therefore, there are numerous research and work analyzing the ECG signals. The state of cardiac health is generally reflected in the shape of ECG waveform and heart rate. It may contain important pointers to the nature of diseases afflicting the heart. Since the biological signals are unstable, this reflection may occur at random in the time scale. And the ECG signal may differ for the same person such that they are different from each other and at the same time similar for different types of heartbeats. In this situation, the disease symptoms may not show up all the time, but would manifest at certain irregular intervals during the day. Therefore, the computer auto analysis of ECG remains the research 
hotspot in medical Engineering. Computer based classifications of the ECG can achieve high accuracy and offer the potential of an affordable mass screening for cardiac abnormalities. Successful classification is achieved by finding the characteristic shapes of the ECG that discriminate effectively between the required diagnostic Categories. Conventionally, a typical heart beat is identified from the ECG and the component waves of the QRS, T and possibly P waves are characterized using measurements such as magnitude, duration and area.

For several years, many researches concern automated analysis of ECG signals and several methods have been developed to increase the accuracy and sensitivity [1-13]. These methods include Wavelet Coefficient, Autoregressive Modeling, RBF Neural Networks, self-organizing map, Rough Sets and fuzzy c-means clustering techniques. In [1], a method of ECG beat recognition using fuzzy hybrid neural network is proposed. In [2], a neuron fuzzy network approach for the ECG-based classification is described. Here, the QRS complex signal is characterized by Hermite polynomials, whose coefficients feed the neuron-fuzzy classifier. In [3], a rule-based rough-set decision system is pressented for the development of an inference engine for disease identification using time-domain features. In [4], a new approach based Particle Swarm Optimization and Support Vector Machines has been proposed for feature selection and classification of cardiac arrhythmias. In [5], the detection of arrhythmia by means of ICA and WT to extract important features is described. A method of ECG Arrhythmia Classification using Modufar Neural Network Model is presented in [6]. In [7], heartbeat classification using feâture selection driyen by database generalization criteria is proposed. Ref. [8] used MLP and RBFN for ECG classification and achieved the classification accuracy of $98 \%$ using MLP and $97 \%$ using RBF. In [9], the authors proposed an arrhythmia recognition based on NSVM-KNN hybrid classifier. An ECG signal processing for abnormalities detection using multi-resolution wavelet transform and ANN classifier is shown in [10].

Although the methods described above could work successfully in recognizing certain types of ECG signals, the recognition rate usually can not be substantially promoted throughout all kinds of ECG signals. In fact, the major of these methods still need artificial assistance at the present. For improving the accuracy, many scholars are trying to look for a more excellen method. In this paper, a rough sets and quantum neural network based classifier is proposed to classify the ECG signal into normal and abnormal classes. The MIT-BIH is one of the most popular and useful databases. It is selected to verify our work. In our work, we first extract feature parameter from the normalization ECG signal with wavelet transform (WT) and then classify these signals with QNN after reducing the feature attribute set using RSalgorithm.

In the following sections, the method of extraction feature from ECG signal and feature reduction based on RS theory will be introduced. Section 3 details the design of the classifier and the experiment results. We state our conclusion in Section 4.

\section{Feature Extraction and Selection}

ECG signal analysis is usually divided into five parts: acquisition of signal, de-noising, feature extraction, feature selection and classification. In this section we will detail feature extraction, selection and classifier design for ECG signal analysis and the procedure designed for this purpose. Figure 1, presents the procedure of the ECG signal analysis. 


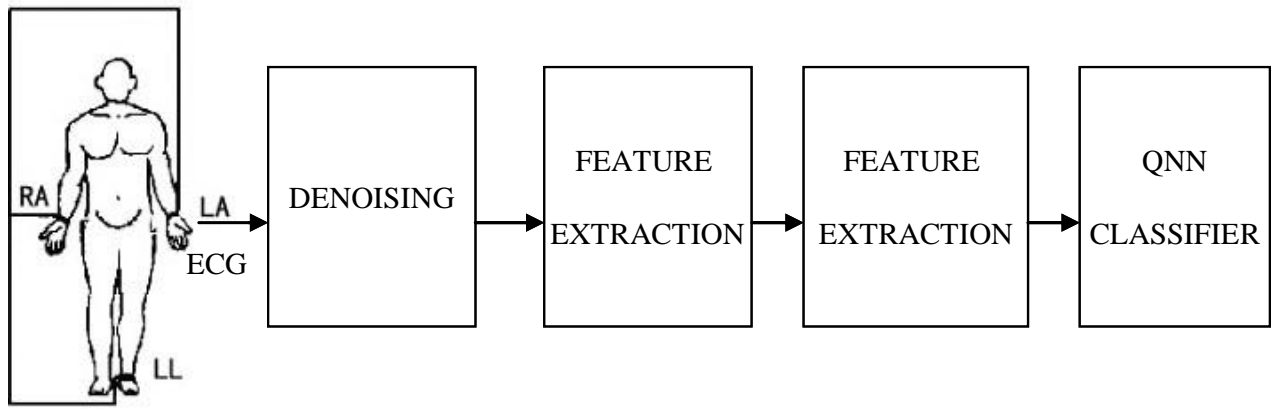

Figure 1. The procedure of the ECG signal analysis

\subsection{Feature Extraction using Wavelet Transform}

The noise of ECG waveform contains power line interference wit 60N, nhuscle electricity and baseline wander. The pretreatment of ECG wave is completed based on a high-pass filter with $0.7 \mathrm{~Hz}$ and low-pass filter with $100 \mathrm{~Hz}$ (see Figures 3 and 4) $[14]$.

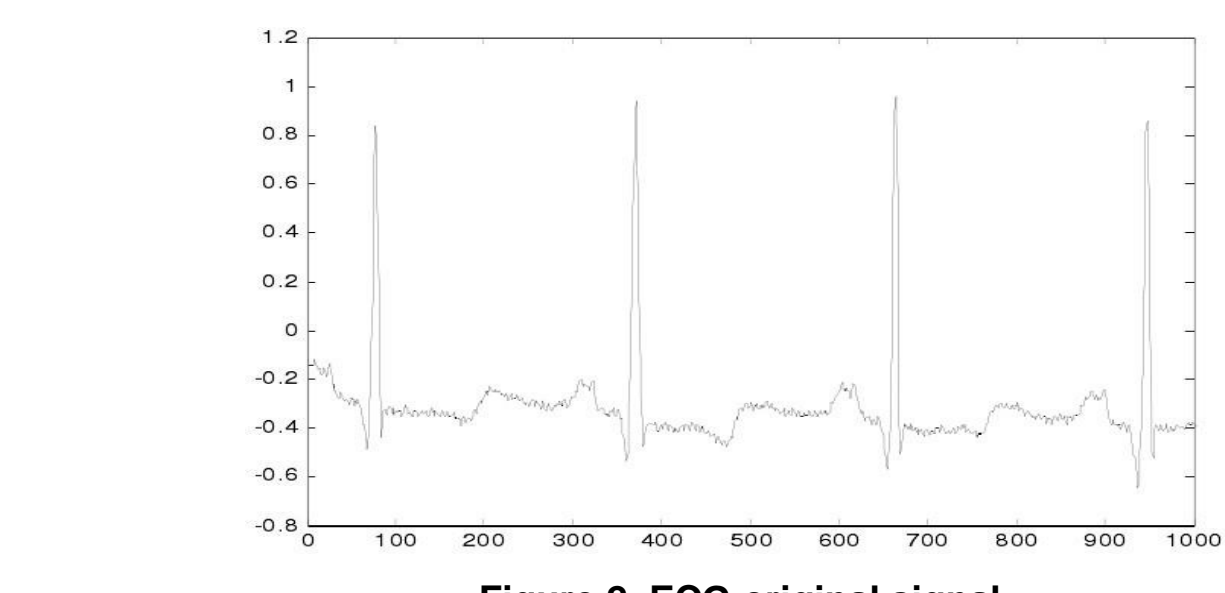

Figure 2. ECG original signal

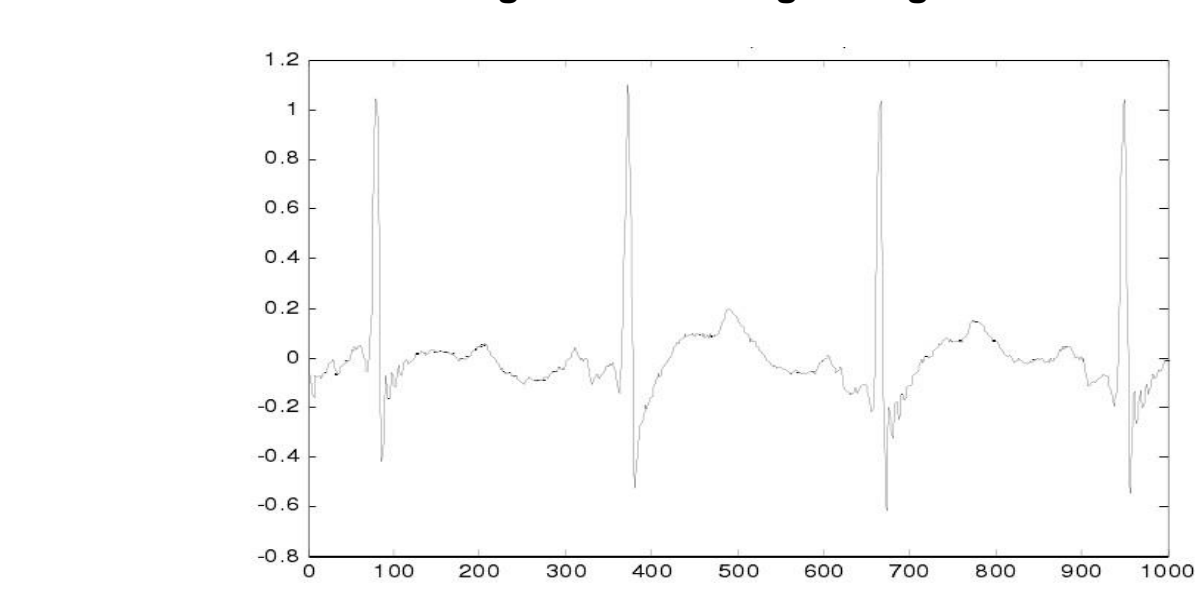

Figure 3. The ECG signal after de-noising 
After the noise elimination, baseline wanders removal and peak detection $(\mathrm{P}, \mathrm{Q}, \mathrm{R}, \mathrm{S}$ and $\mathrm{T})$ [15], it is necessary to extract the feature of the ECG waveform in order to use it in the classifier design stage of ECG signal analysis.

The ECG is a non-stationary time signal. For such signal, time frequency representations are desirable. The frequency characteristics as well as the temporal behavior can be described with wavelet transform. It can decompose the signals into various components depending upon the application and reassemble these components into the original signal without any information loss. So it is a powerful technique to analyze the physiological signals such as ECG and EEG. Basically wavelet transform is the convolution operation of the subject signal $f(t)$ and the wavelet function $\psi(t)$. The discrete wavelet transform is expressed as,

$$
X_{j, k}=\int_{-\infty}^{+\infty} f(t) \psi_{j, k}(t) d t
$$

The approximation coefficient of the signal $f(t)$ is represented as,

$$
A_{j, k}=\int_{-\infty}^{+\infty} f(t) \phi_{j, k}(t) d t
$$

Where $\phi(t)$ is scaling function, $j$ and $k$ are scale and location respectively. For a range of scale $n$, the original signal $f(t)$ under discrete wavelet transform can be represented as,

$$
f(t)=f_{n}(t)+\sum_{j=1}^{n} d_{j}(t)
$$

Where $f_{n}(t)$ is mean signal approximation and is given by,

$$
f_{n}(t)=A_{n, k} \phi_{n, k}(\tau) ?
$$

and $d_{j}(t)$ is detail signal approximation in scale $j$.

Thus in discrete wavelet domain, multi-resolution analysis can be performed. For each of ECG signals, there are 28 wavelet based features have been obtained [16]. Figure 4 is a single heartbeat illustrating the majer landmarks within an ECG.

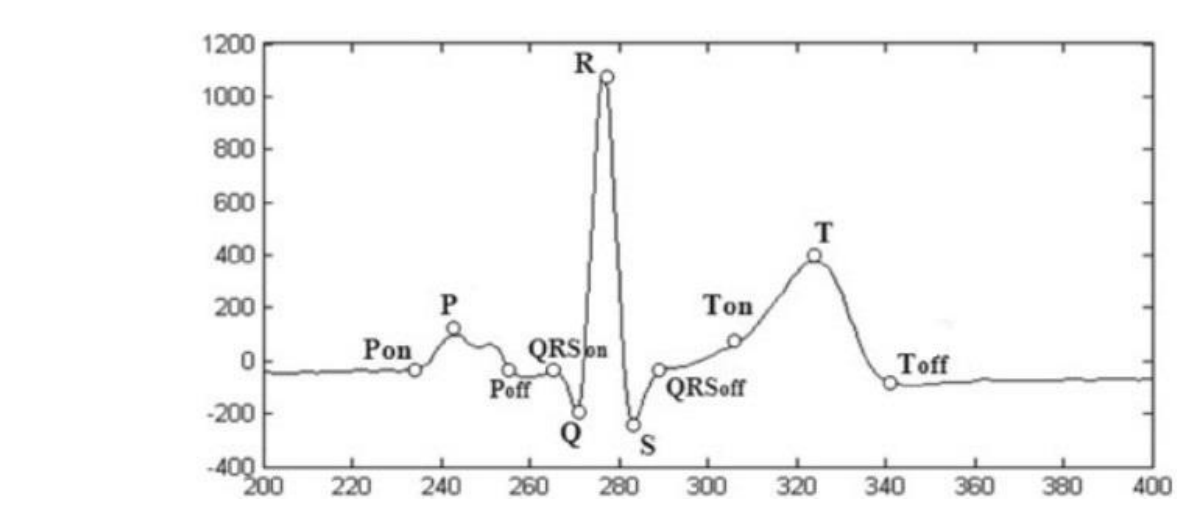

Figure 4. A single heartbeat illustrating with the major landmarks

These features encompass 19 temporal features (distances between fiducial points), 6 amplitude features (values of P, Q, R, S, T peaks) and 3 angle features, as shown in Figure 5. Due to the ECG signal is the continuous time signal. We use first order differencing method to discretize the continuous attribute. 


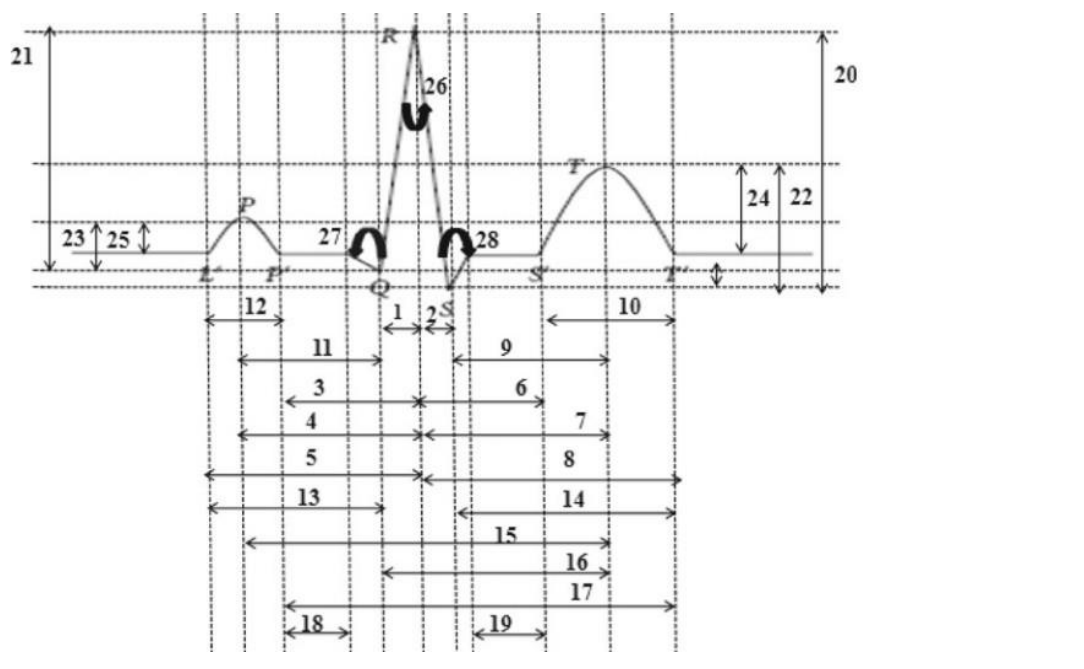

Figure 5. 28 Fiducial features in a single heartbeat waveform

\subsection{Feature Reduction Based on RS}

The theory of rough sets is an importan method in knowledge discovery field. It is a mathematical approach which is developed as a method for extracting information from decision table through a set of reduction and decision rules. It has attracted a great deal of attention recently in field of data pining and feature selection. The attribute reduction based on RS theory is applied as a preprocessor before classification so that we could delete redundant attributes and inegnsistent objects from decision table but remain efficient information lossless. By this, method, we may reduce the dimension of data and speed up the processing time.

We assume that the readers are fandiliar with the RS theory. Here, we will not narrate the basic concept of it.

Input: decision table $S=(U, C, F, D, V)$

Output: the attribute reduction result $\operatorname{red}(C)$ of the decision table $S$.

\section{Step 1: (1nitiating)}

Calcufate the degree of dependency of conditional attributes $r_{C}(D)=\frac{\operatorname{card}\left(P O S_{C}(D)\right)}{\operatorname{card}(U)}$, if $r_{C}(D)=1$, the decision table is consistent and we go to the next step.

Step 2: (Eliminating redundant attribute)

Compute every degree of dependency of $C-a_{j}$ respectively, $a_{j} \in\left\{a_{1}, a_{2}, \mathrm{~L}, a_{m}\right\}$. For each $a_{j} \in A$, if $r_{C-a_{j}}(D) \neq 1$, end.

Then $a_{j}$ is indispensable in the set $C$, we could obtain the reduction set $\operatorname{red}(C)=C$. 
Step 3: If $a_{i} \in A, r_{C-a_{I}}(D)=1$, then $a_{i}$ is dispensable in the set $C$, we note $B_{i}=C-a_{i}$.

Step 4: (Redefine the attribute $\operatorname{set} C$ )

Redefine $C=B_{i}=C-a_{i}$ and return step 3 .

Repeat step 3(from $i=1$ to $m$ ) until the decision is inconsistent, i.e., $r_{C-a_{j}}(D) \neq 1$.

Step 5: (Return and output reduction) $\operatorname{red}(C)=\mathrm{U} B_{i}$.

Thus we remove redundant input attributes from feature data decision table $S=(U, C, F, D, V)$ using RS method. $U$ is the data set of samples, the flinite set $C$ is the feature attributes, $D=\{0,1\}$. Where 0 is signed to normal samples, 1 is signed to abnormal samples.

\section{The Design of ECG Classifier}

\subsection{Learning algorithm of quantum neural networks}

Quantum Neural Network (QNN) is Q youthful and energetic science built upon the combination of quantum computing and artificial neural network. It also combines the advantages of neural modelling and fuzzy theoretic principles. The professor S. Kak firstly presented the concept of quantum neurar computation in 1995 [17]. In 1998, the first systematic and deep examination of quantum artificial neural network was performed by $\mathrm{T}$. Menneer in his Ph.D. dissertation [18]. There are many literatures about the model and its application of the QNN $[19-22]$.

Be similar with classic neural networks, QNN has an inherently fuzzy architecture which can decompose the sample information into discrete levels of certainty or uncertainty [22]. The transfer function of the quantum neuron has the ability to form graded partitions instead of crisp linear partitions of the feature space. If the feature vector lies at the boundary between overlapping classes, the QNN will assign it partially to all related classes. If no certainty exists, QNN will assign it to the corresponding class. One possibility of obtaining this kind of transfer function is to take the superposition of $n s$ sigmoid functions, each shiffed by quantum interval $\theta_{s}(s=1,2, \mathrm{~L}, n s)$, where $n s$ is the number of levels or sigmoid in the hidden unit.

The output of the multi-layer excitation function of hidden neurons in QNN can be written as:

$$
\frac{1}{n s} \sum_{s=1}^{n s} f\left[\beta *\left(W^{T} X-\theta_{s}\right)\right]
$$

where $f(x)=1 /(1+\exp (-x)), W$ is the network weights vector, $X$ is the network input vector, $\beta$ is the slope factor. Figure 6 is the activation functions of quantum neural net work. 

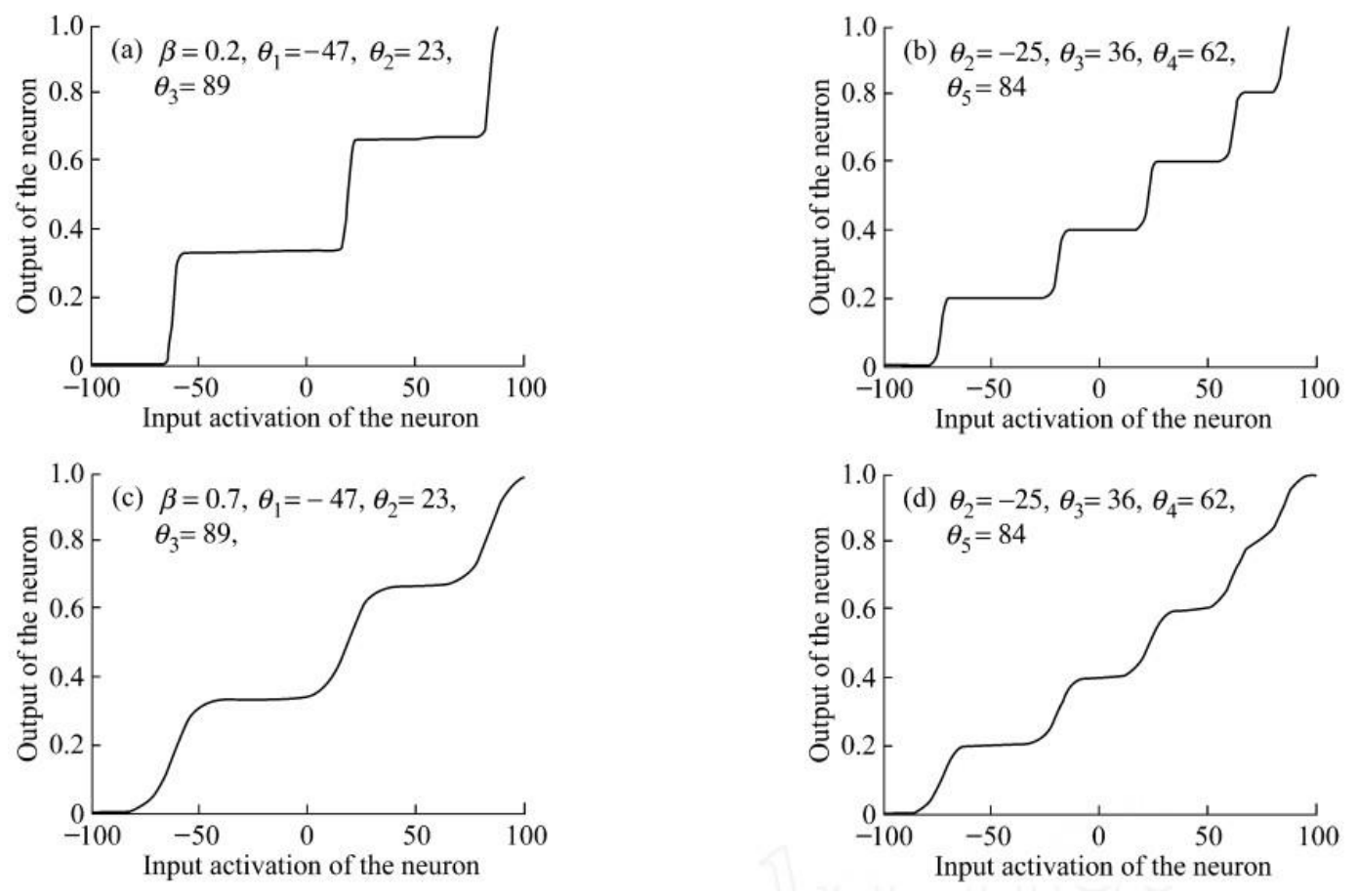

Figure 6. Activation fonctions of quantum neural net work

The gradient descent method is used to trainthe QNN of multi-layer excitation function. In each training cycle, the training algorithm revises both the connection weight between the different level neuron and quantum intervals of the hidden layer. When the synaptic weights have been obtained, hidden neurons quantum intervals can be learned by minimizing the class-conditional variances at the outpyts of the hidden units [24, 25].

The variance of the output of the $i$ th hidden unit for class $C_{m}$ is

$$
\delta_{i, m}^{2}=\sum_{x_{k} \in C_{m}}\left(\left\langle O_{i, m}\right\rangle-O_{i, k}\right)^{2}
$$

where $O_{i, k}$ is in the input of the ith neuron in hidden layer when the input vector is $x_{k}$, $\left\langle O_{i, m}\right\rangle=\frac{1}{C C_{m}} \sum_{k} \in C_{m} O_{i, k},\left|C_{m}\right|$ is the cardinal number of $C_{m}, m$ is pattern class number.

By minnimizing $\delta_{i, m}^{2}$, we can get the update equation for $\theta_{i, s}$ as follows. For each hidden unit $i$ and its $s$ th quantum level $(s=1,2, \mathrm{~L}, n s)$,

$$
\Delta \theta_{i, s}=\alpha \frac{\beta}{n s} \sum_{m=1}^{n_{0}} \sum_{x_{k} \in C_{m}}\left(\left\langle O_{i, m}\right\rangle-O_{i, k}\right) *\left(\left\langle v_{i, m, \mathrm{~s}}\right\rangle-v_{i, k, \mathrm{~s}}\right)
$$

In the forum, $\alpha$ is the learning rate; $n_{0}$ is the number of output layer nodes, namely the total class number; $n_{s}$ is quantum interval of layers, $x_{k} \in x_{m}$ means that among all samples belong to the class $C_{m} ;\left\langle v_{i, m, \mathrm{~s}}\right\rangle$ and $v_{i, k, \mathrm{~s}}$ obtained by the following two equations: 


$$
\begin{aligned}
& \left\langle v_{i, m, \mathrm{~s}}\right\rangle=\frac{1}{\left|C_{m}\right|} \sum_{x_{k} \in C_{m}} v_{i, \mathrm{k}, \mathrm{s}} \\
& v_{i, \mathrm{k}, \mathrm{s}}=O_{i, \mathrm{k}, \mathrm{s}} *\left(1-O_{i, \mathrm{k}, \mathrm{s}}\right) .
\end{aligned}
$$

where $O_{i, \mathrm{k}, \mathrm{s}}=f\left[\beta *\left(w^{T} x_{k}-\theta_{s}\right)\right]$ and $f(x)=1 /(1+\exp (-x))$. When the input vector is $x_{k}$, it is the output of $s$ of the ith neuron in the hidden layer.

\subsection{Experiment results and Comparison}

MIT-BIH arrhythmia database is used as source of experimental data. The palt waveform signals $T_{100}, T_{105}, T_{106}, T_{108}, T_{111}, T_{112}, T_{217}, T_{219}, T_{220}, T_{221}$ are selected from MIT-BIH (http://physionet.org /physiobank/database/). For each signal, we randomly selected 40 wave forms to analyze. And we randomly selected 20 wave forms to train the hetwork. In Table 1, the performances of the proposed method are compared with other algorithms [26].

Table 1. Comparison of the classification result

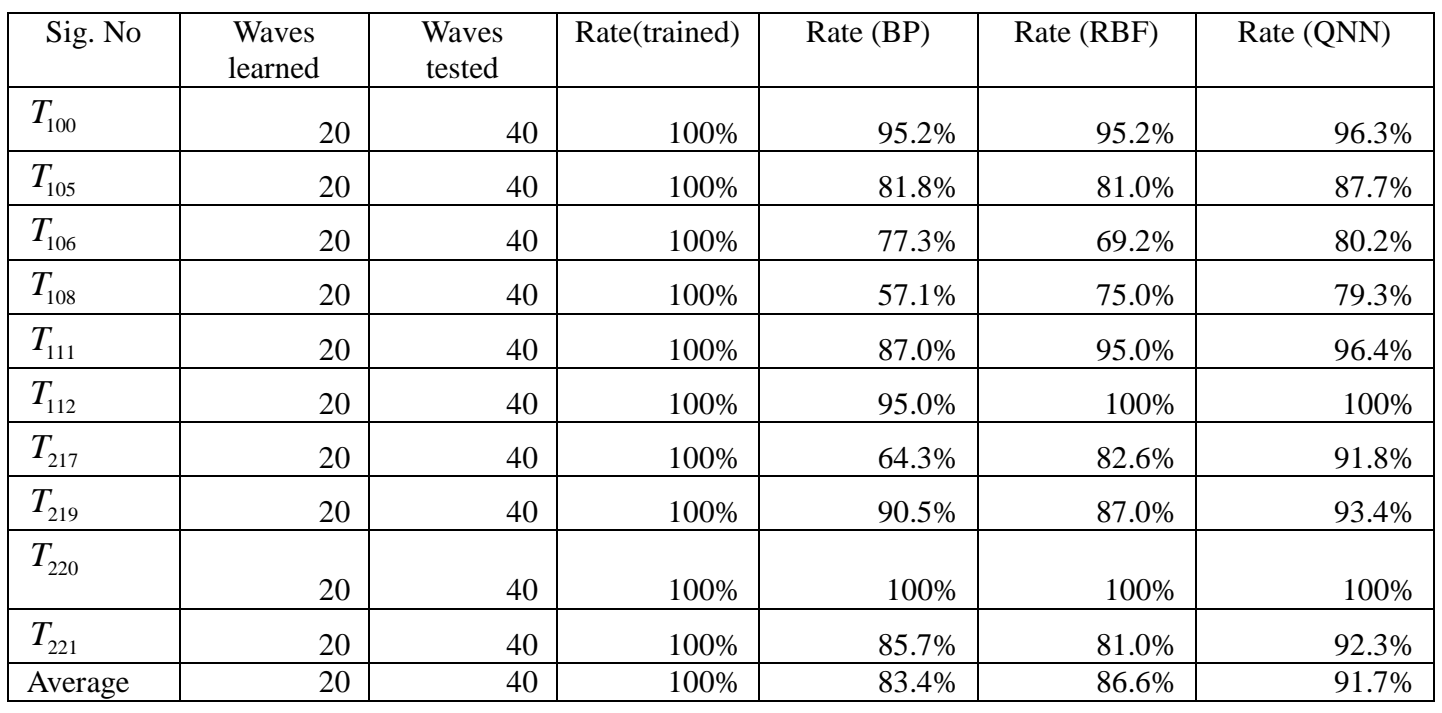

\section{Conclusions}

In this paper, an ECG classification method with RS and Quantum Neural Networks is presented. As discussed in the previous section, we firstly preprocess the ECG signal with a filter design. After the noise elimination, feature extraction and attribute reduction are conducted. Then a classifier based on QNN is proposed. The major conclusions of this work indicate that the QNN is superior in most respects to BP and RBF network, and attribute reduction based on rough sets theory enhances classification performance. The experiment result shows that the present method could effectively use to recognize ECG signal. 


\section{References}

[1] S. Osowski and T. H. Linh, "ECG beat recognition using fuzzy hybrid neural network", IEEE Trans. Biomed. Eng., vol. 48, no. 11, (2001), pp. 1265-1271.

[2] T. H. Linh, S. Osowski and M. L. Stodoloski, "On-line heart beat recognition using Hermite polynomials and neuron-fuzzy network”, IEEE Trans. Instrum. Meas., vol. 52, no. 4, (2003), pp. 1224-1231.

[3] S. Mitra, M. Mitra and B. B. Chaudhuri, "A rough set-based inference engine for ECG classification", IEEE Trans. Instrum. Meas., vol. 55, no. 6, (2006), pp. 2198-2206.

[4] F. Melgani and Y. Bazi, "Classification of electrocardiogram signals with support vector machines and particle swarm optimization”, IEEE Trans. Inf. Technol. Biomedicine, vol. 12, no. 5, (2008), pp. 667-677.

[5] A. Azemi, V. R. Sabzevari, M. Khademi, H. Gholizadeh, A. Kiani and Z. Dastgheib, "Intelligent,Arrhythmia Detection and Classification Using ICA", Proceedings of the 28th IEEE, EMBS Annual Tnterndtional Conference, New York City, USA, (2006) August 30-September 3.

[6] M. Shivajirao Jadhav, L. Sanjay Nalbalwar and A. Ashok Ghatol, "ECG Arrhythmia classification using modular neural network model”, IEEE EMBS Conference on Biomedical Engineering \& Sćences (IECBES 2010), Kuala Lumpur, Malaysia, (2010) November 30-December 2.

[7] M. Llamedo and J. P. Martınez, "Heartbeat classification using leature selection driven by database generalization criteria", IEEE Trans. Biomedical Eng., vol. 58, nю.3,(2011).

[8] V. Mai, I. Khalil and C. Meli, "ECG biometric using multilayer perceptron and radial basis function neural networks", 33rd Annual International Conference of the IEEE EMBS Boston, Massachusetts USA, (2011) August 30-September 3.

[9] M. R. Homaeinezhad, S. A. Atyabi, E. Tavakkoli, AH. N. Toosi, A Gharfari and R. Ebrahimpour, "ECG arrhythmia recognition via a neuro-SVM-KNN hybrid classifier withyirtual QRS image-based geometrical features", Expert Syst. Appl., vol. 39, (2012), pp. 2047-2058.

[10] H. Mohan Rai, A. Trivedi and S. Shukl. "ECG signal processing for abnormalities detection using multi-resolution wavelet transform and Aftificial Neural Network classifier", Measurement, vol. 46, (2013), pp. 3238-3246.

[11] Y. C. Yeh, W. J. Wang and C. W.Chidu, "A novel fuzzy c means method for classifying heartbeat cases from ECG signals", Measurement, vol. 43, (2010), pp..1542-1555.

[12] M. M. Tantawi, K. Revett, A. Salem and M. F. Tolba, "Fiducial feature reduction analysis for electrocardiogram (ECG) based biometric recognition", Journal of Intelligent Information Systems, vol. 40, (2013), pp. 17-39.

[13] A. De Gaetano, S. Panunz,, F. Rinaldi, A. Risi and M. Sciandrone, "A patient adaptable ECG beat classifier based on neural networks", Applied Mathomatics and Computation, vol. 213, (2009), pp. 243-249.

[14] D. Liu, "Research on Quantum Neural Network Model and Its Application to ECG Classification", Master's thesis, Nanjing Unlversity of Posts and Telecommunications, (2012).

[15] S. Pal and M. Mitra, "Detection of ECG characteristic points using multi-resolution wavelet analysis based selective coefficient method" Measurement, vol. 43, (2010), pp. 255-261.

[16] M. M. Tantawi, K. Revett, M. F. Tolba and S. Abdel-Badeeh, "An evaluation of the generalisability and applicability of the PhysioNet electrocardiogram (ECG) repository as test cases for ECG-based biometrics", International Journal of Cognitive Biometrics. Inderscience, vol. 1, no. 1, (2012), pp. 66-97.

[17] S. Kak, "On Quantum Neural Computing", Information Sciences, vol. 83, (1995), pp. 143-160.

[18] T. Menneer, "Quantum artificial neural networks”, PhD thesis, Univ. of Exeter, UK, (1998).

[19] R. G Zhow and Q. L. Ding, "Quantum M-P Neural Network, Int J Theor Phys., vol. 46, (2007), pp. 3209-3215.

[20] 1. Aljazaery, A. A. Ali and M. H. Abdulridha, "Classification of Electroencephalograph (EEG) Signals Using Quantum Neural Network”, Signal Processing: An International Journal (SPIJ), vol. 4, no. 6, (2011), pp. 329-337.

[21] J. Zhou, Q. Gan, A. Krzyzak and Y. C. Suen, "Recognition of handwritten numerals by Quantum Neural Network with fuzzy features", International Journal on Document Analysis and Recognition, vol. 2, (1999), pp. 30-36.

[22] Y. Xu, X. F. Zhang and H. C. Gai, "Quantum Neural Networks for Face Recognition Classifier”, Advanced in Control Engineering and Information Science, Procedia Engineering, vol. 15, (2011), pp. 1319 - 1323.

[23] G. Purushothaman and B. N. Karayiannis, "Quantum Neural Networks (QNN's): Inherently fuzzy feed forward neural networks", IEEE Trans. on Neural Networks, vol. 8, no. 3, (1997), pp. 679-693.

[24] O. R. Duda and E. P. Hart, "Pattern Classification and Scene Analysis", New York: John Wiley, (1973).

[25] P. Gopathy, B. Nicolaos, and N. B. Karayiannnis, "Quantum neural network computes entanglement", IEEE Transactions on Neural Networks, vol. 8, no. 3, (1997), pp. 679-693. 
International Journal of Multimedia and Ubiquitous Engineering

Vol.9, No.2 (2014)

[26] J. Feng, "ECG Classification Based on Feature-extraction and Neural Network", Master's thesis, Sichuan Normal University, (2005).

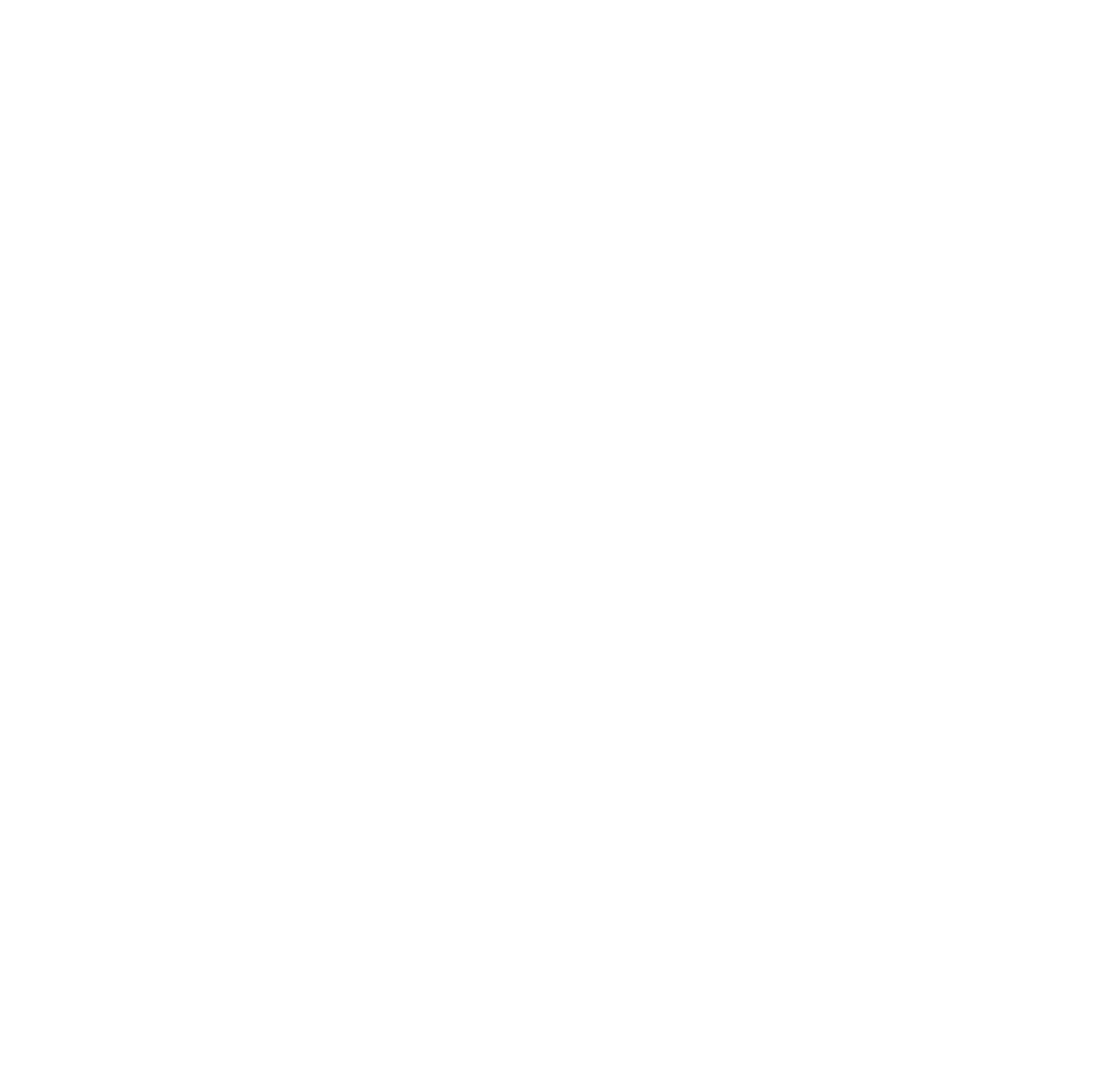

